\title{
PERCEPÇÃO DE IDOSOS POBRES COM ALTERAÇÕES COGNITIVAS SOBRE FUNCIONALIDADE FAMILIAR
}

\author{
Ariene Angelini dos Santos², Sofia Cristina Iost Pavarini³, Elizabeth Joan Barham ${ }^{4}$
}

\footnotetext{
1 Trabalho extraído da dissertação - Idosos com alterações cognitivas: um estudo sobre a funcionalidade familiar em contexto de pobreza, apresentada ao Programa de Pós-Graduação em Enfermagem da Universidade Federal de São Carlos (UFSCar), em 2009, com apoio financeiro da CAPES e FAPESP.

${ }^{2}$ Mestre em Enfermagem. São Paulo, Brasil. E-mail: arieneangelini@yahoo.com.br

${ }^{3}$ Doutora em Educação. Professora associado do Departamento de Enfermagem da UFSCar. São Paulo, Brasil. E-mail: sofia@ ufscar.br

${ }^{4}$ Doutora em Psicologia Social e Aplicada. Professora associado do Departamento de Psicologia da UFSCar. São Paulo, Brasil. E-mail: lisa@ufscar.br
}

RESUMO: O objetivo foi avaliar a influência do gênero e da idade nas percepções de relações familiares entre idosos com alterações cognitivas que moravam em contextos de pobreza. Trata-se de estudo transversal com abordagem quantitativa. A funcionalidade familiar foi avaliada utilizando o instrumento Apgar de Família, durante entrevista domiciliar com 45 idosos, com alterações cognitivas, que moravam em contextos de pobreza. Os resultados mostram que $62 \%$ das mulheres e $95 \%$ dos homens avaliaram a funcionalidade de sua família como "boa", $21 \%$ das mulheres e $5 \%$ dos homens apontaram moderada disfunção e apenas as mulheres $(17 \%)$ apontaram elevada disfunção familiar. Estes resultados foram significativamente diferentes, segundo o teste de Mann-Whitney $(Z=-2,559 ; p=0,01)$. Não houve correlação significativa, pelo coeficiente de Spearman, entre idade e funcionamento familiar $(p>0,05)$. Assim, no desenvolvimento de serviços públicos de cuidado ao idoso com alterações cognitivas, é importante considerar diferenças ligadas ao gênero, na percepção de relações familiares.

DESCRITORES: Idoso. Demência. Relações familiares. Identidade de gênero. Grupos etários.

\section{PERCEPTIONS ON FUNCTIONING FAMILY FROM POOR BRAZILIAN ELDERLY WITH COGNITIVE IMPAIRMENTS}

\begin{abstract}
The objective this cross-sectional study with a quantitative approach was to evaluate the influence of gender and age on perceptions of family relations, among elderly Brazilians with cognitive impairments living in impoverished neighborhoods. Family functioning was assessed using the Family Apgar Instrument, during in-home interviews with 45 elderly Brazilians with cognitive impairments living in impoverished neighborhoods. The results indicate that $62 \%$ of the women and $95 \%$ of the men rated their family functioning as "good", $21 \%$ of the women and $5 \%$ of the men reported moderate family dysfunction, and only women $(17 \%)$ reported high levels of dysfunction. These results were significantly different, based on the Mann-Whitney test $(Z=-2.559$; $p=0.01)$. The correlation between age and family functioning was not significant, based on Spearman's coefficient $(p>0.05)$. Thus, in the development of public services for elderly with cognitive impairments it is important to consider gender-related differences in perceptions of family relationships.
\end{abstract}

DESCRIPTORS: Aged. Dementia. Family relations. Gender identity. Age groups.

\section{PERCEPCIÓN DE ANCIANOS POBRES CON ALTERACIONES COGNITIVAS SOBRE FUNCIONALIDAD FAMILIAR}

\begin{abstract}
RESUMEN: El objetivo fue evaluar la influencia del género y edad en las percepciones de relaciones familiares, entre ancianos con alteraciones cognitivas que vivían en contextos de pobreza. Se trata de un estudio transversal con enfoque cuantitativo. La funcionalidad familiar fue evaluada utilizando el instrumento Apgar de familia, durante una entrevista domiciliar con 45 ancianos con alteraciones cognitivas que vivían en contextos de pobreza. Los resultados revelan que el $62 \%$ de las mujeres y el $95 \%$ de los hombres evaluaron la funcionalidad de su familia "buena"; el 21\% de las mujeres y el 5\% de los hombres presentaron moderada disfunción y solamente las mujeres (17\%) señalaron elevada disfunción familiar. Estos resultados fueron significativamente diferentes, según el test de MannWhitney $(Z=-2,559 ; p=0,01)$. No hubo correlación significativa, por el coeficiente de Spearman, entre edad y funcionalidad familiar $(p>0,05)$. Así que, en el desarrollo de servicios públicos de atención al anciano con alteraciones cognitivas, es importante considerar diferencias con respecto al género en la percepción de las relaciones familiares..
\end{abstract}

DESCRIPTORES: Anciano. Demencia. Relaciones familiares. Identidad de género. Grupos por edad. 


\section{INTRODUÇÃO}

Com o envelhecimento, há um aumento na prevalência de demências, a qual duplica a cada cinco anos, a partir dos 60 anos de idade. ${ }^{1} \mathrm{O}$ cuidado destinado a pessoas com alterações cognitivas, geralmente, é assumido pela família, sendo considerada a fonte primária de apoio. ${ }^{2}$

O termo família pode ser definido por duas ou mais pessoas que são ligadas por íntimas associações, recursos e valores e é reconhecida quando os membros consideram os elementos que a constituem como família. ${ }^{3}$

As famílias passaram por profundas transformações nas últimas décadas e como consequência tornaram-se menores e com um maior número de idosos em sua composição. Os membros mais velhos e frágeis estão vivendo por mais tempo, o que significa uma convivência familiar intergeracional mais prolongada. ${ }^{4} \mathrm{O}$ fato das famílias terem se tornado menores nos dias atuais, seja pela redução do número de filhos ou pela maior instabilidade dos casamentos, traz a necessidade de se rever o suporte familiar disponível ao idoso, visto que esse apoio pode ficar limitado. ${ }^{5}$

Embora a estrutura familiar mais frequentemente encontrada para idosos morando na comunidade seja a família multigeracional, não há certeza de que as famílias estejam aptas para assumir o papel de cuidadora do idoso. ${ }^{6} \mathrm{~A}$ avaliação da funcionalidade familiar, enquanto um recurso terapêutico, é uma ferramenta importante no auxílio às equipes assistenciais. ${ }^{7}$ Isso pode ser realizado com um instrumento denominado Apgar de Família. ${ }^{8}$

O Apgar de Família (Family Apgar), desenvolvido por Smilkstein, em 1978, é um instrumento composto por cinco questões. Estas questões permitem medir a satisfação dos membros da família em relação a cinco componentes considerados básicos na unidade e funcionalidade de qualquer família: adaptação, companheirismo, desenvolvimento, afetividade e capacidade resolutiva. $\mathrm{O}$ Apgar de Família foi validado, traduzido e adaptado para nossa cultura, utilizando para isso a metodologia de adaptação transcultural. ${ }^{8,9}$

Esse instrumento permite identificar as percepções individuais dos valores da família como um recurso psicossocial (escore elevado) ou como um suporte social deficitário e possível fator estressor (escore baixo). ${ }^{9}$ Uma adequada funcionalidade familiar sugere que essa família esteja apta a absorver e lidar com situações de crise. Esse tipo de avaliação pode auxiliar os profissionais da área de saúde a planejarem suas intervenções. ${ }^{7}$

Estudos mostram os resultados da aplicação do instrumento Apgar de Família com idosos. ${ }^{10-11}$ Em Cuba, foram entrevistados 70 idosos, com 60 anos de idade ou mais, não institucionalizados e sem alterações cognitivas. Os dados mostraram que, com relação à faixa etária, não houve resultados estatisticamente significativos, predominando uma boa funcionalidade familiar no grupo de idosos com 65 a 69 anos. Quanto à estrutura familiar, os idosos que moravam em contextos familiares multigeracionais afirmaram ter uma moderada funcionalidade familiar. Nas famílias nucleares foi verificada boa funcionalidade familiar. ${ }^{10}$

Um estudo realizado na Venezuela teve como sujeitos 140 idosos que eram atendidos em um ambulatório, dos quais 70 faziam parte do grupo controle (tinham outros problemas de saúde e não apresentavam depressão) e os outros 70 idosos eram portadores de depressão. Como resultados, notaram um alto índice de disfunção familiar nos idosos depressivos (61\%) e, dentro deste percentual, $30 \%$ era considerada como disfunção severa. ${ }^{11}$

Em relação ao gênero, a literatura brasileira sobre o envolvimento de homens e mulheres no provimento de cuidados para com familiares indica que a maioria dos cuidadores de idosos são mulheres. ${ }^{12}$ Desta forma, em geral, as mulheres possuem mais experiência no papel de cuidador familiar do que os homens, podendo ter critérios diferentes e até mais exigentes para avaliar a qualidade dos cuidados, além de expectativas mais altas em relação aos cuidados que gostariam de receber. Há diferenças na forma como homens e mulheres pensam sobre questões tais como o casamento, o papel parental e o trabalho. ${ }^{13} \mathrm{Com}$ base no exposto, espera-se que as percepções de funcionamento familiar também devam ser afetadas pelo gênero do respondente.

Estudos mostram que a idade avançada pode levar a uma situação de maior dependência dos idosos e, portanto uma demanda maior por cuidados de longa duração. Em geral esse cuidado pode levar a uma situação de estresse e sobrecarga do cuidador. ${ }^{12,14}$ Desta forma, esperase que os respondentes mais idosos avaliem o funcionamento familiar de forma mais negativa do que os menos idosos.

Este trabalho teve por objetivo verificar a influência do gênero e da idade nos resultados do Apgar de família de idosos com alterações 
cognitivas, cadastrados em Unidades de Saúde da Família (USFs), que moram em contextos de alta vulnerabilidade social.

\section{MÉTODO}

Trata-se de um estudo descritivo, transversal, baseado nos pressupostos da pesquisa quantitativa. Foi realizado em um município do interior paulista. Segundo o censo de 2000, a população da cidade era de 192.998 habitantes, 11\% dos quais apresentando 60 anos ou mais. ${ }^{15}$ Em 2007, esse município contava com 12 equipes de Saúde da Família. O número de idosos cadastrados nas USFs neste mesmo ano era de aproximadamente 4700 pessoas, correspondendo a $8,7 \%$ do total de pessoas de todas as idades cadastradas.

Neste estudo foi considerado o Índice Paulista de Vulnerabilidade Social (IPVS) do setor censitário da USF onde o idoso estava cadastrado. O IPVS classifica os setores censitários do Estado de São Paulo segundo níveis de vulnerabilidade social a que estão sujeitos os seus residentes variando de um (nenhuma vulnerabilidade) a seis (vulnerabilidade muito alta). ${ }^{16}$ Foram incluídas as unidades com IPVS 5 e 6, ou seja, as unidades localizadas em contextos de alta e muito alta vulnerabilidade social.

Foram sujeitos desta pesquisa 45 pessoas com idade a partir de 60 anos ( $\mathrm{F}=24$ e $\mathrm{M}=21)$, cadastradas e avaliadas em USFs de regiões com alto índice de vulnerabilidade social desse município. Os critérios de inclusão dos sujeitos foram: ter 60 anos de idade ou mais; estar cadastrado em uma unidade com alto índice de vulnerabilidade social (IPVS 5 ou 6); apresentar resultado no Mini Exame do Estado Mental, abaixo da nota de corte, de acordo com o grau de escolaridade (indicativo de alteração cognitiva); não possuir comprometimentos graves de linguagem ou compreensão avaliados por meio de uma entrevista inicial; assinar o Termo de Consentimento Livre e Esclarecido.

Foi realizado um estudo piloto a fim de verificar se havia dificuldades na aplicação do instrumento e calcular o tamanho amostral. Nesse estudo piloto foram avaliados cinco idosos de cada uma das USFs com IPVS 5 e 6. Considerou-se um nível de confiança de $95 \%$ e uma precisão de $10 \%$. Foi realizada alocação proporcional para distribuir a amostra pelos estratos, sendo 18 idosos relativos à USF com IPVS 6 e 27 idosos relatiovs à USF com IPVS 5, totalizando 45 idosos.

Todos os cuidados éticos que regem pesquisas com seres humanos foram observados, segun- do a Resolução 196/96. O projeto foi aprovado pelo Comitê de Ética em Pesquisa da Universidade onde a pesquisa foi realizada (Parecer 253/2008). Os participantes foram orientados sobre os propósitos e anonimato do estudo. A coleta de dados teve início após a leitura e assinatura do Termo de Consentimento Livre e Esclarecido, tanto pelo idoso portador de alterações cognitivas, quanto pelo cuidador familiar responsável.

Foram realizadas visitas domiciliares previamente agendadas, nas residências dos 45 idosos. Os dados foram coletados no período de agosto a outubro de 2008. A coleta de dados consistiu de uma entrevista estruturada com os idosos, utilizando-se um instrumento denominado Apgar de Família para avaliar a funcionalidade familiar desses idosos.

Como referido anteriormente, o Apgar de Família permite a avaliação de cinco componentes, com questão às quais são atribuídos escores que variam de zero a quatro. No final, esses escores são somados, resultando num escore total cujo valor numérico relaciona-se diretamente com a condição da funcionalidade familiar (boa funcionalidade, moderada ou alta disfuncionalidade). ${ }^{9}$

A análise estatística empregada foi não-paramétrica. Para comparar os resultados de homens e mulheres foi utilizado o teste de Mann-Whitney, pois foram comparados dois grupos independentes. Para determinar a correlação entre idade e o escore Apgar e seus itens, foi utilizada a correlação de Spearman. O nível de significância adotado foi de $5 \%(p \leq 0,05)$.

\section{RESULTADOS}

\section{Apgar de Família e gênero}

Na comparação do Apgar de Família de homens e mulheres, pelo teste de Mann-Whitney, verificou-se que os escores apresentados foram significativamente diferentes $(\mathrm{p}=0,010)$, conforme mostra a Tabela 1.

$\mathrm{Na}$ avaliação dos cinco itens que compõem o Apgar de Família em relação ao sexo masculino e feminino, foram verificados, por meio do Teste de Mann-Whitney, que as questões relativas à adaptação (questão a), ao companheirismo (questão b) e à afetividade (questão d) foram estatisticamente significativas e nas questões referentes ao desenvolvimento (questão c) e à capacidade resolutiva (questão e), os resultados não foram estatisticamente significantes. 
Tabela 1 - Análise estatística comparativa de cada dimensão do Apgar de Família para homens e mulheres, de acordo com o teste de Mann-Whitney. São Carlos - SP, 2009

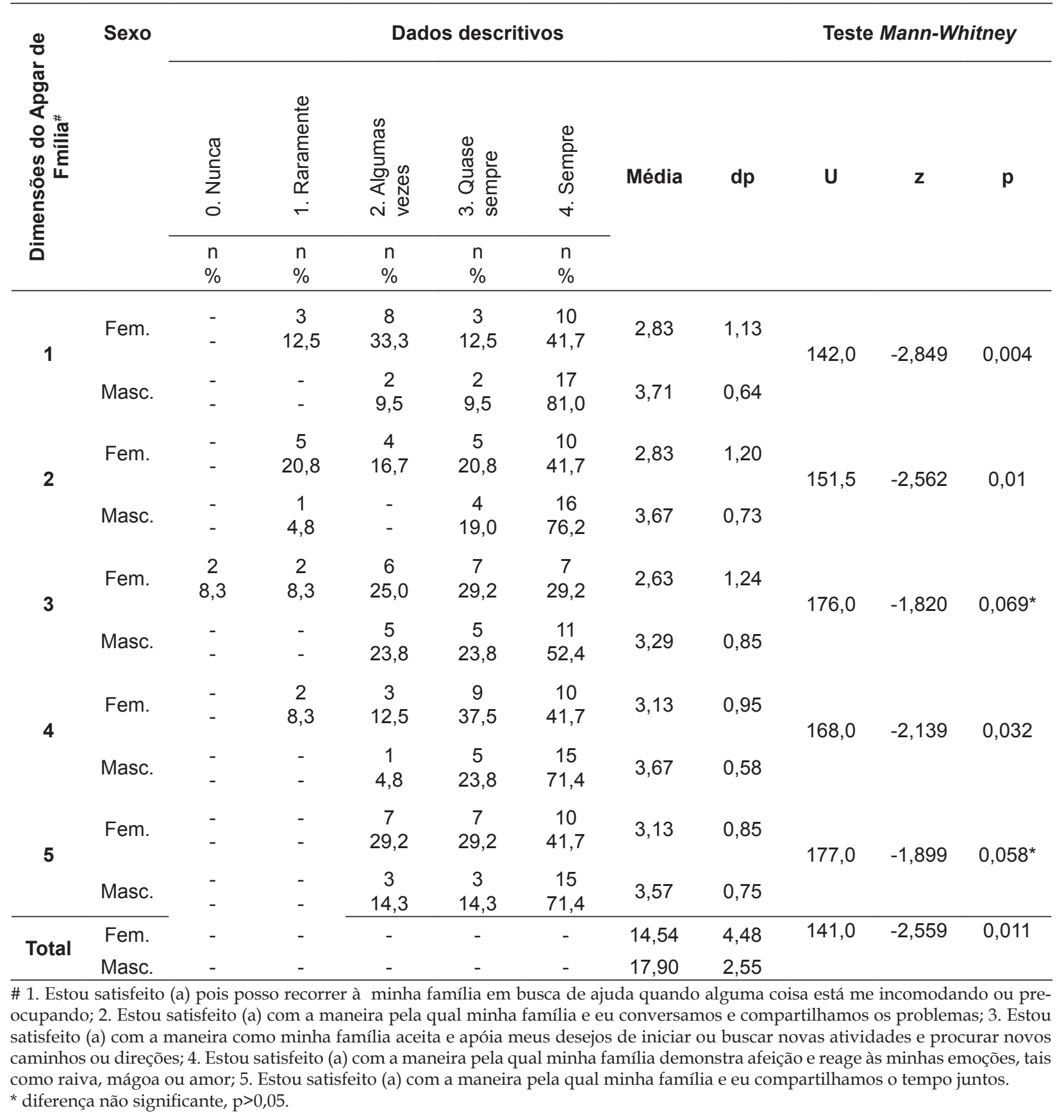

A análise estatística comparativa de cada domínio do Apgar de Família para homens e mulheres será descrita a seguir.

A primeira questão - "Estou satisfeito (a), pois posso recorrer à minha família em busca de ajuda quando alguma coisa está me incomodando ou preocupando" - refere-se ao componente Adaptação. Nessa questão, nenhum homem e $12,5 \%$ das mulheres afirmaram raramente estar satisfeitos ao recorrerem à família em busca de ajuda, 33\% das mulheres e 9,5\% dos homens responderam que algumas vezes estavam satisfeitos, $12,5 \%$ das mulheres e $9,5 \%$ dos homens afirmaram quase sempre estar satisfeitos e $42 \%$ das mulheres e $81 \%$ dos homens relataram estar sempre satisfeitos por poderem recorrer à família em busca de ajuda.

A segunda questão do Apgar de Família - "Estou satisfeito (a) com a maneira pela qual minha família e eu conversamos e compartilhamos 
os problemas" - refere-se ao componente Companheirismo. Nesse item, 21\% das mulheres e 5\% dos homens relataram raramente estar satisfeitos com a maneira pela qual ocorriam as comunicações familiares e a resolução de problemas, $17 \%$ das mulheres e $0 \%$ dos homens afirmaram algumas vezes estar satisfeitos, $21 \%$ das mulheres e $19 \%$ dos homens quase sempre satisfeitos e $41 \%$ das mulheres e $76 \%$ dos homens sempre satisfeitos com a reciprocidade das comunicações familiares e resolução de problemas.

O terceiro componente (Desenvolvimento) - "Estou satisfeito (a) com a maneira pela qual minha família aceita e apóia meus desejos de iniciar ou buscar novas atividades e procurar novos caminhos ou direções" - foi aquele em que houve maior satisfação dos homens em relação às mulheres. Nessa questão, $52 \%$ dos homens e $29 \%$ das mulheres afirmaram estar sempre satisfeitos com o apoio oferecido pela família, porém essas diferenças não chegaram a ser significativas $(p>0,050)$.

O quarto componente, Afetividade, referese à questão - "Estou satisfeito (a) com a maneira pela qual minha família demonstra afeição e reage às minhas emoções, tais como raiva, mágoa ou amor". Nesse item, os dados revelam que $8 \%$ das mulheres e $0 \%$ dos homens raramente estavam satisfeitos com as interações emocionais no contexto familiar, $12,5 \%$ das mulheres e $5 \%$ dos homens algumas vezes estavam satisfeitos, 37,5\% das mulheres e $24 \%$ dos homens afirmaram quase sempre estar satisfeitos e $42 \%$ das mulheres e $71 \%$ dos homens relataram sempre estar satisfeitos com a afetividade da família.

A questão - "Estou satisfeito (a) com a maneira pela qual minha família e eu compartilhamos o tempo juntos" está relacionada ao quinto componente, isto é, Capacidade Resolutiva. Nessa questão, houve maior satisfação dos homens em relação às mulheres, porém essas diferenças não chegaram a ser significativas $(p>0,050)$. Embora os percentuais de respostas "sempre" de homens e mulheres sejam muito próximos aos da questão anterior, a questão d) traz que $8 \%$ das mulheres responderam "raramente", o que torna as diferenças entre as distribuições maiores.

A categoria "sempre satisfeito" foi acima de $50 \%$ para os cinco componentes avaliados em relação aos participantes do sexo masculino desse estudo: adaptação (81\%), companheirismo (76\%), desenvolvimento (52\%), afetividade (71\%) e capacidade resolutiva (72\%). Entre as mulheres, a categoria "raramente satisfeitas" foi bastante citada em quatro domínios avaliados pelo Apgar, ou seja, em relação à adaptação, 12,5\% afirmavam estar raramente satisfeitas, companheirismo (21\%), desenvolvimento $(8,5 \%)$ e afetividade $(8 \%)$.

Finalmente, totalizando e agrupando os escores dos entrevistados em relação à funcionalidade familiar, verificou-se que $62 \%$ das mulheres e $95 \%$ dos homens apresentaram boa funcionalidade familiar, $21 \%$ das mulheres e $5 \%$ dos homens moderada disfunção familiar e apenas as mulheres apresentaram elevada disfunção familiar, perfazendo $17 \%$, conforme mostra a Figura 1.

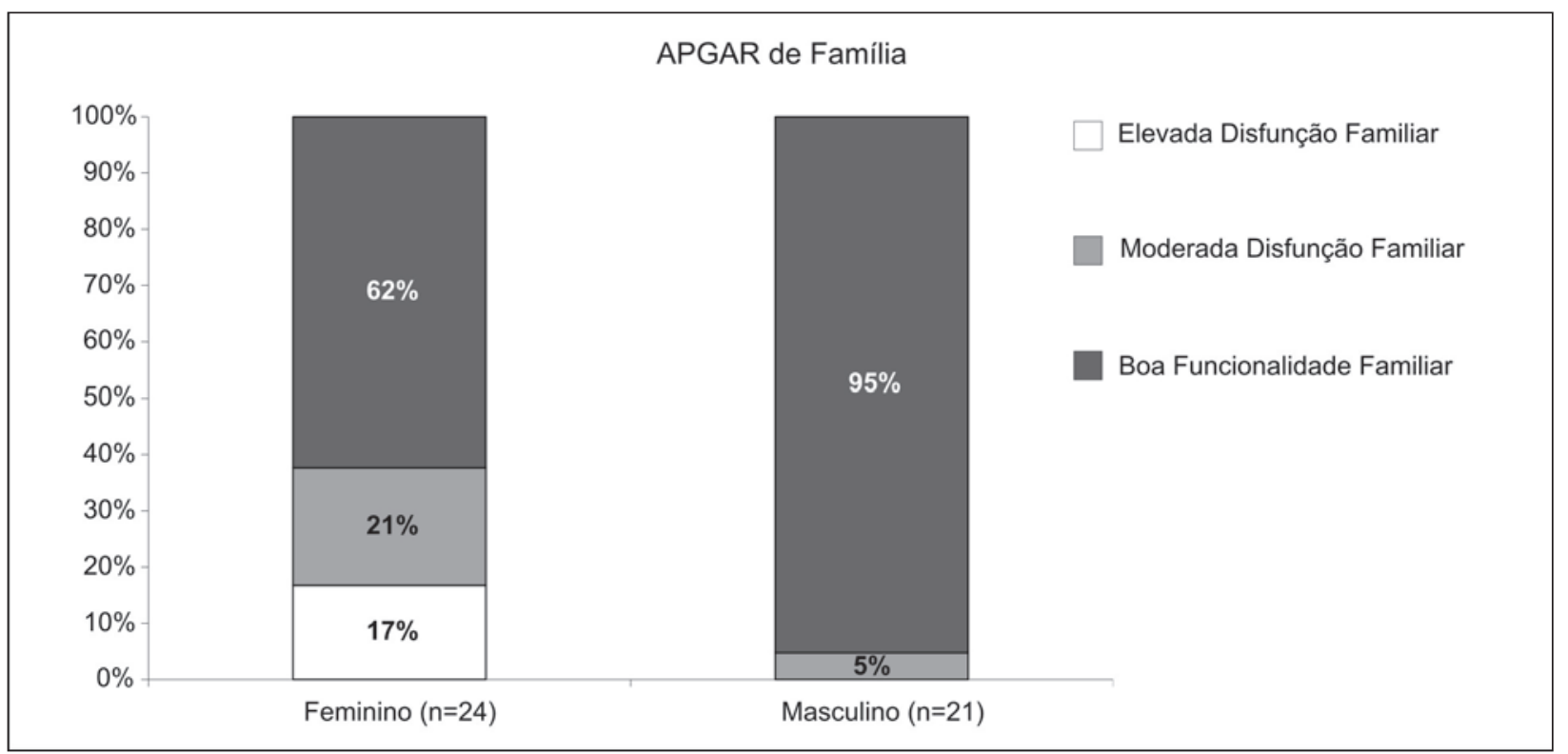

Figura 1 - Porcentagem dos idosos em relação à funcionalidade familiar segundo gênero. São Carlos - SP, 2009. 


\section{Apgar de Família e idade}

Na avaliação do grau de correlação do Apgar de Família com a idade dos idosos, pelo coeficien- te de correlação de Spearman, verificou-se que as correlações não foram significativas $(p>0,050)$, conforme mostram a Figura 2 e a Tabela 2.

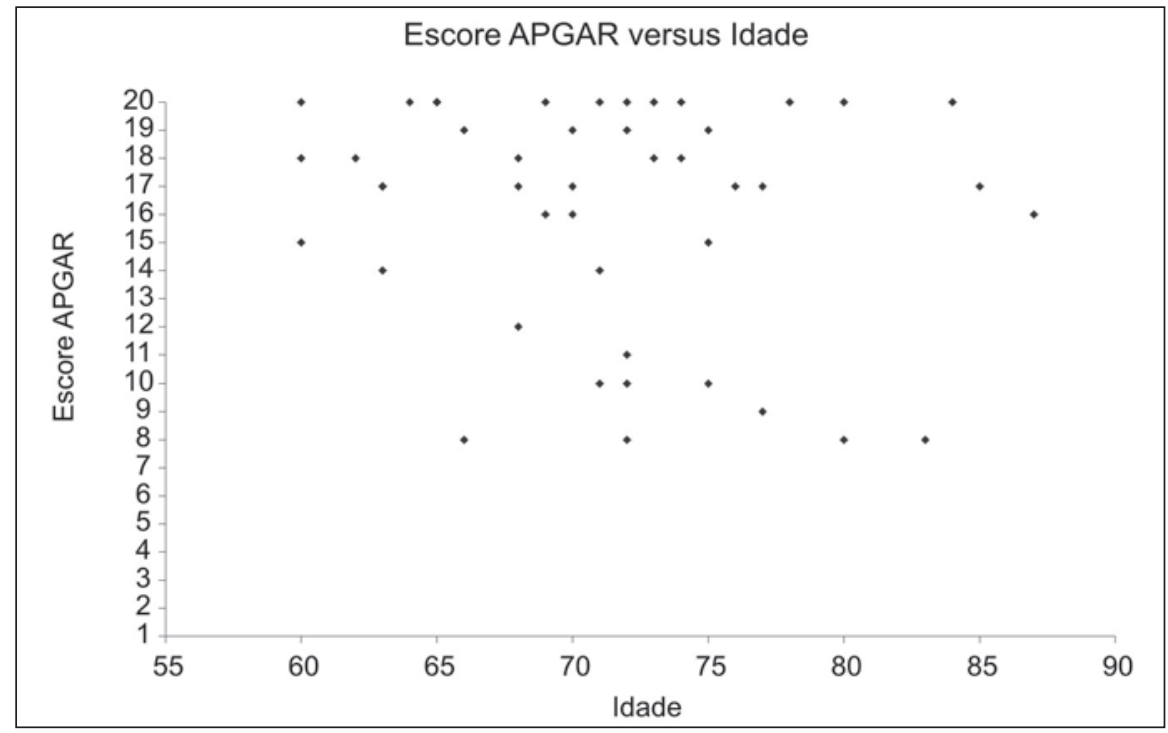

Figura 2 - Relação entre o escore Apgar de Família e idade dos sujeitos. São Carlos - SP, 2009.

Tabela 2 - Correlação entre o escore de cada dimensão do Apgar de Família e a idade dos sujeitos. São Carlos - SP, 2009

\begin{tabular}{lcc}
\hline Correlação de Spearman & & Idade \\
\hline Escore APGAR & $\mathrm{r}$ & $-0,118$ \\
Questão a) & $\mathrm{p}$ & 0,441 \\
& $\mathrm{r}$ & $-0,237$ \\
Questão b) & $\mathrm{p}$ & 0,117 \\
& $\mathrm{r}$ & $-0,095$ \\
Questão c) & $\mathrm{p}$ & 0,535 \\
& $\mathrm{r}$ & $-0,168$ \\
Questão d) & $\mathrm{p}$ & 0,271 \\
& $\mathrm{r}$ & $-0,050$ \\
Questão e) & $\mathrm{p}$ & 0,747 \\
& $\mathrm{r}$ & 0,006 \\
$\mathrm{n}=45$ & $\mathrm{p}$ & 0,968 \\
\hline
\end{tabular}

\section{DISCUSSÃO}

Acredita-se ser fundamental para o planejamento assistencial adequado ao idoso, a compreensão de seu contexto familiar, o que implica no conhecimento das questões que envolvem a formação e a dinâmica de funcionamento das famílias em geral. ${ }^{17}$ Essa avaliação da funcionalidade familiar pode ser feita por meio do instrumento denominado Apgar de Família.

O Apgar de Família demonstrou ser um instrumento útil na avaliação da funcionalidade familiar de idosos pobres com alterações cognitivas, podendo ser usado para uma atuação mais eficaz dos profissionais de saúde e, especialmente, do enfermeiro de USFs, por trabalhar diretamente com famílias, de modo a direcionar as intervenções à realidade do idoso pobre.

Há muitas lacunas ainda na literatura sobre a funcionalidade familiar de idosos, fato que sugere o investimento em futuros estudos.

O predomínio de boa funcionalidade familiar entre idosos foi também encontrado em outras pesquisas. Um estudo realizado com 93 pessoas acima de 60 anos de idade, de ambos os sexos, independentes e participantes de um movimento de alfabetização de adultos de uma cidade do interior de São Paulo, mostrou que houve predominância de entrevistados do sexo feminino e que $87,1 \%$ dos idosos apresentaram boa funcionalidade familiar. Do restante, $8,6 \%$ apresentou moderada disfunção familiar e 4,3\% elevada disfunção familiar. ${ }^{18}$ 
No Chile, foi feito um trabalho no qual houve predomínio de mulheres (68\%) e da faixa etária dos 70 aos 79 anos (48,3\%), obtendo como resultado que $84 \%$ desses idosos afirmaram ter um bom funcionamento familiar, descrevendo como altamente positivo o funcionamento de sua família. ${ }^{19}$

Boa funcionalidade familiar ou leve disfunção familiar foram identificadas em $75 \%$ dos participantes de uma pesquisa realizada na Venezuela, com 95 famílias. ${ }^{20}$

Um estudo realizado em São Paulo, que comparou o resultado do Apgar em idosos brasileiros independentes, dependentes e seus cuidadores, mostrou que os valores do Apgar de Família foram mais elevados entre os idosos independentes, seguido pelos idosos dependentes e por último pelos cuidadores. Valores do Apgar de Família inferiores nos grupos de idosos dependentes e no grupo dos cuidadores de idosos mostram que o funcionamento da família no cumprimento de suas funções enquanto unidade familiar está mais comprometido quando comparados com os valores obtidos no grupo dos idosos independentes. ${ }^{9}$

Nossos achados mostraram não haver correlação significativa entre a faixa etária e o Apgar de Família dos idosos participantes. Esses dados estão de acordo com o estudo realizado em São Paulo, ${ }^{9}$ no qual também não houve correlação significativa entre faixa etária e Apgar de Família dos idosos entrevistados.

No presente estudo, tratando-se de gênero, os resultados foram estatisticamente significativos, os quais mostraram que as mulheres estão mais insatisfeitas do que os homens em relação à funcionalidade familiar. Esses dados são corroborados pela pesquisa realizada em São Paulo. ${ }^{9}$

Em relação ao gênero, as pessoas encaram naturalmente a divisão feita entre o que é trabalho feminino e masculino. Ao homem cabe o público, o poder, a manutenção financeira da família, traçar diretrizes e metas, dirigir e comandar os altos cargos nas hierarquias funcionais, enquanto que as mulheres são destinadas à responsabilidade pelos filhos, estruturação do lar, cuidado dos idosos, ou seja, tudo que se relaciona à esfera doméstica. ${ }^{21}$

Dessa forma, a pesquisa feita em São Paulo aponta que as mulheres exercem papéis importantes na dinâmica familiar e o fato delas se encontrarem em condições mais limitadas, ou seja, com alterações cognitivas ou dependentes funcionalmente, fazem com que elas não desempenhem suas tarefas domésticas de modo integral, e assim, vejam o funcionamento de sua família de forma negativa, isto é, de modo disfuncional. ${ }^{9}$

Supõe-se que, em condições de disfunção familiar, as famílias poderiam ter a sua capacidade assistencial prejudicada e assim não conseguiriam prover adequadamente o atendimento sistemático das necessidades de cuidados de entes queridos idosos, podendo desta forma, interferir na qualidade de vida desses idosos. ${ }^{22}$

Outro fenômeno importante a ser discutido trata-se do predomínio de pessoas do sexo feminino em tarefas do cuidado. O predomínio de mulheres cuidadoras reforça o papel social da mulher, historicamente determinado, ou seja, quem realiza as tarefas de cuidar em família é uma mulher, uma vez que prestar cuidado é secularmente uma atribuição feminina, quer seja esposa, filha ou irmã. Entretanto, a maioria relata não receber ajuda de outras pessoas no desempenho desta atividade, tornando-se sobrecarregadas. Estudos mostram que essa sobrecarga está relacionada à falta de ajuda por parte dos familiares. A cuidadora, ao assumir sozinha os cuidados do idoso no domicílio, manifesta frequentemente seu desconforto e sentimento de solidão, quando não sente apoio de outros membros da família, o que poderia interferir no cuidado prestado aos idosos. ${ }^{23}$ Isso pode estar influenciando de maneira direta a maior insatisfação das mulheres em relação à funcionalidade familiar, pois como as mulheres possuem mais experiência no papel de cuidadora, são mais exigentes para avaliar a qualidade dos cuidados recebidos e a dinâmica familiar.

\section{CONCLUSÕES}

Não houve correlação significativa entre a idade e o Apgar de Família de idosos com alterações cognitivas, cadastrados em USFs e que moram em contextos de alta vulnerabilidade social.

Houve influência do gênero nos resultados do Apgar de Família dos idosos estudados. Os dados nos mostram que, para cada domínio do Apgar de Família, as mulheres apresentaram escores mais baixos em relação aos homens, indicando moderada disfunção familiar, o que sugere que a família pode não estar apta totalmente para cuidar desses idosos.

Esses dados poderão auxiliar na implantação de uma linha de pesquisa a idosos com alterações cognitivas no município no âmbito da Estratégia de Saúde da Família, especialmente pelo fato de que o cuidado destinado a idosos no Brasil é 
realizado na maioria das vezes por familiares do sexo feminino.

O conhecimento da funcionalidade familiar é importante para o desenvolvimento de estratégias de assistência domiciliária mais efetiva, capaz de assistir às demandas crescentes dos idosos e de suas famílias, buscando o equilíbrio familiar, visto que o suporte familiar contribui de maneira significativa para a manutenção e a integridade física e psicológica do indivíduo.

\section{AGRADECIMENTOS}

Agradecemos o apoio financeiro da Fundação de Amparo à Pesquisa do Estado de São Paulo e à Coordenação de Aperfeiçoamento de Pessoal de Nível Superior.

\section{REFERÊNCIAS}

1. Lopes MA, Bottino CMC. Prevalence of dementia in several regions of the world: analysis of epidemiologic studies from 1994 to 2000. Arq Neuropsiquiatr. 2002 Mar; 60(1):61-9.

2. Caldas CP. Cuidando do idoso que vivencia uma síndrome demencial: a família como cliente da enfermagem. Texto Contexto Enferm. 2001 MaiAgo; 10(2):68-93.

3. Bomar PJ. Promoting health in families: applying family research and theory to nursing practice. $3^{\mathrm{a}}$ ed. Pensylvânia (US): Elsevier; 2004.

4. Souza RFS, Skubs T, Brêtas ACP. Envelhecimento e família: uma nova perspectiva para o cuidado de enfermagem. Rev Bras Enferm. 2007 Mai-Jun; 60(3):263-7.

5. Alves AM. Os idosos, as redes de relações sociais e as relações familiares. In: Neri AL, organizadora. Idosos no Brasil: vivências, desafios e expectativas na terceira idade. São Paulo (SP): Editora Fundação Perseu Abramo; 2007. p.125-39.

6. Pavarini SCI, Barham EJ, Mendiondo MSZ, Filizola CLA, Petrilli Filho JF, Santos AA. Family and social vulnerability: a study with octogenarians. Rev Latino-am Enfermagem. 2009 Mai-Jun; 17(3):374-9.

7. Freitas EV. Tratado de geriatria e gerontologia. $2^{\mathrm{a}}$ ed. Rio de Janeiro (RJ): Guanabara Koogan; 2006.

8. Smilkstein G. The family APGAR a proposal for a family function test and its use by physicians. J Fam Pract. 1978 Jun; 6(6):1231-9.

9. Duarte YAO. Família: rede de suporte ou fator estressor: a ótica de idosos e cuidadores familiares [tese]. São Paulo (SP): Universidade de São Paulo. Programa de Pós-Graduação em Enfermagem; 2001.
10. Díaz Tabares O, Soler Quintana ML, García Capote M. El apgar familiar en ancianos conviventes. Rev Cubana Med Gen Integr [online]. 1998 Nov-Dic [acesso 2008 Abr 03]; 14(6). Disponível em http:/ / scielo.sld.cu/pdf/mgi/v14n6/mgi07698.pdf

11. Benevides O, Reyes-Ortiz C, Herrera JA. Prevalencia de sintomas depressivos en ancianos. Med Fam (Caracas) 1999 Jul-Dic; 7(2):52-9.

12. Amendola F, Oliveira MAC, Alvarenga MRM. Qualidade de vida dos cuidadores de pacientes dependentes no programa de saúde da família. Texto Contexto Enferm. 2008 Abr-Jun; 17(2):266-72.

13. Gravena AC. Retornando ao trabalho após o nascimento de um filho: percepções de professoras sobre sua experiência [dissertação]. São Carlos (SP): Universidade Federal de São Carlos. Programa de Pós Graduação em Educação Especial; 2006.

14. Luzardo AR, Gorini MIPC, Silva APS. Características de idosos com doença de Alzheimer e seus cuidadores: uma série de casos em um serviço de neurogeriatria. Texto Contexto Enferm. 2006 OutDez; 15(4):587-94.

15. IBGE [online]. Censo 2000. [acesso 2008 Mai 08]. Disponível em: http://www.ibge.gov.br

16. Fundação Sistema Estadual de Análise de Dados SEADE [online]. São Paulo-SP. Índice Paulista de Vulnerabilidade Social - IPVS. Espaços e dimensões da pobreza nos municípios do Estado de São Paulo. [acesso 2007 Set 14]. Disponível em: http:/ / www. seade.gov.br/produtos/ipvs/pdf/oipvs.pdf

17. Torres GV, Reis LA, Reis LA, Fernandes MH, Alves GS, Sampaio LS, et al. Funcionalidade familiar de idosos dependentes residentes em domicílios. Aval Psicol. 2009 Dez; 8(3):415-23.

18. Pavarini SCI, Tonon FL, Silva JMC, Mendiondo MSZ, Barham EJ, Filizola CLA. Quem irá empurrar minha cadeira de rodas? A escolha do cuidador familiar do idoso. Rev Eletr Enferm [online]. 2006[acesso 2007 Out 10]; 8(3). Disponível em http:/ / www.fen.ufg. $\mathrm{br} /$ revista/revista8_3/v8n3a03.htm

19. Mercedes Zavala G, Daisy Vidal G, Manuel Castro S, Pilar Quiroga, Gonzalo Klassen P. Funcionamiento social del adulto mayor. Cienc Enferm [online]. 2006 Dic [acesso 2008 Abr 03]; 12(2). Disponível em: http: / / www.scielo.cl/scielo.php?pid=S071795532006000200007\&script=sci_arttext

20. Ferrer M. Valores y funcionabilidad familiar en habitantes del sector San Jose Coro-Falcón. Med Fam Caracas. 2003 Jul-Dic; 11(2):20-7.

21. Villela WV, Arilha M. Sexualidade, gênero e direitos sexuais e reprodutivos. In: Berquó E, organizadora. Sexo e vida: panorama da saúde reprodutiva no Brasil. Campinas (SP): Ed. Unicamp; 2003. p.95-150. 
22. Silveira TM, Caldas CP, Carneiro TF. Cuidando de idosos altamente dependentes na comunidade: um estudo sobre cuidadores familiares principais. Cad Saúde Pública. 2006 Ago; 22(8):1629-38.
23. Santos AA, Pavarini SCI. Perfil dos cuidadores de idosos com alterações cognitivas em diferentes contextos de vulnerabilidade social. Rev Gaúcha Enferm. 2010 Mar; 31(1):115-22. 